\title{
Visible-light photocatalytic efficiencies and anti-photocorrosion behavior of CdS/graphene nanocomposites: Evaluation using methylene blue degradation
}

\author{
Jiajia Yan, Kun Wang *, Hui Xu, Jing Qian, Wei Liu, Xingwang Yang, Huaming Li \\ Key Laboratory of Modern Agriculture Equipment and Technology, School of Chemistry and Chemical Engineering, Jiangsu University, Zhenjiang \\ 212013, Jiangsu, China
}

\section{A R T I C L E I N F O}

\section{Article history:}

Received 21 February 2013

Accepted 1 April 2013

Published 20 October 2013

Keywords:

CdS nanocrystal

Graphene nanocomposite

Anti-photocorrosion

Photocatalysis

Methylene blue degradation

\begin{abstract}
A B S T R A C T
A series of CdS nanocrystals/graphene (CdS/GR) nanocomposites with various graphene contents were prepared. Their photocatalytic efficiencies and anti-photocorrosion behavior were studied using methylene blue degradation under visible-light irradiation. The results showed that the introduction of graphene improved the migration efficiency of photogenerated carriers and inhibited charge carrier recombination. The photocatalytic efficiencies of the as-prepared CdS/GR nanocomposites were influenced by the graphene content, achieving a maximum at a graphene content of 4.6 wt $\%$ (denoted by CdS/GR-4.6\%). The anti-photocorrosion behavior of the CdS/GR-4.6\% nanocomposite was further investigated using a photoelectrochemical method and X-ray diffraction analysis. The photocorrosion of CdS nanocrystals was effectively suppressed after the introduction of graphene sheets. Compared with bare CdS nanocrystals, the photocurrent of the CdS/GR-4.6\% nanocomposites increased 2.3-fold.
\end{abstract}

(c) 2013, Dalian Institute of Chemical Physics, Chinese Academy of Sciences. Published by Elsevier B.V. All rights reserved.

\section{Introduction}

In recent years, graphene has been recognized as an ideal high-performance photocatalyst carrier or promoter because of its unique atom-thick two-dimensional structure, excellent transparency, high specific surface area, excellent electron mobility, and high chemical stability [1,2]. Various graphene-based semiconductor nanocomposites coupled with suitable semiconductor nanocrystals (NCs) have been designed and prepared, and used in photocatalysis applications, including nonselective processes for the degradation of pollutants [3], selective transformations in organic synthesis [4], and water splitting to provide clean hydrogen energy [5]. Many previous studies have proven that the photocatalytic efficiencies of var- ious graphene-based semiconductor nanocomposites were significantly improved by the introduction of graphene sheets $[6,7]$. As is generally known, good stability after catalytic reactions is an important index in evaluating catalytic performance because of the stringent ecological and economic demands for sustainability [8]. The rapid deactivation of photocatalysts is an unavoidable issue in most cases, and this seriously limits the applications of photocatalysts. However, a literature survey showed that previous research on graphene-based photocatalysts focused on enhancing photocatalytic activity and selectivity, and catalyst recovery and anti-photocorrosion behavior were not seriously investigated. It is therefore still necessary to investigate the photostabilities of graphene-based nanocomposites to enable their use in practical applications.

\footnotetext{
*Corresponding author. Tel: +86-511-88791800; Fax: +86-511-88791708; E-mail: wangkun@ujs.edu.cn This work was supported by the National Natural Science Foundation of China $(21175061,21007021)$, the Key Laboratory of Modern Agriculture Equipment and Technology (NZ201109), and the China Postdoctoral Science Foundation (2012M520998). DOI: 10.1016/S1872-2067(12)60677-9 | http://www.sciencedirect.com/science/journal/18722067 | Chin. J. Catal.,Vol. 34, No. 10, October 2013
} 
CdS is an important semiconductor photocatalyst, and it can potentially be applied in pollutant photodegradation [9] and water splitting to produce clean hydrogen energy [10]. The band gap of $\mathrm{CdS}(2.42 \mathrm{eV})$ is narrower than that of $\mathrm{TiO}_{2}(3.2$ $\mathrm{eV}$ ), which facilitates the use of visible light, and this makes CdS a competitive candidate as a photocatalyst. Unfortunately, previous studies $[9,10]$ have proven that CdS has a low separation efficiency of photogenerated electron-hole pairs and is particularly prone to photocorrosion, which is an inherent disadvantage of $\mathrm{CdS}$ photocatalysts and is fatal for its cyclic operation and in environmental applications. To improve the photoactivities and photostabilities of semiconductor photocatalysts, some efforts have been devoted to producing CdS-based nanocomposites by coupling with another semiconductor, a polymer matrix, or carbon nanotubes [9,11-13]. In the resulting $\mathrm{CdS} / \mathrm{TiO}_{2}$ [11], $\mathrm{CdS} / \mathrm{Bi}_{2} \mathrm{~S}_{3}$ [12], CdS/polyaniline [9], or CdS/ carbon nanotube nanocomposites [13], the coupled materials acted as acceptors for photogenerated electrons, promoting charge separation and leaving too few holes on CdS to cause photocorrosion, leading to enhancement of photoreaction efficiency and significant inhibition of photocorrosion. The photoexcited charges were effectively separated and recombination was delayed as a result of the increased photocatalytic activity and suppression of photocorrosion.

In recent years, CdS/graphene (CdS/GR) nanocomposites have been prepared and used as photocatalysts in various applications, including photodegradation of pollutants [6], water splitting to produce clean hydrogen energy [5,14], and selective organic transformations [15], and the high photocatalytic activities of CdS/GR nanocomposites have been seriously investigated in these previous studies. To the best of our knowledge, the anti-photocorrosion and photostability behavior of CdS/GR nanocomposites have not yet been explored. In this study, we prepared a series of CdS/GR nanocomposites with different graphene contents and investigated their photocatalytic efficiencies and anti-photocorrosion behavior under visible-light irradiation. In the photocatalysis reaction system, methylene blue (MB) was selected as the model dye; MB is an important thiazine and is extensively used as a colorant for paper, cotton, silk, and leather [16]. Ultraviolet-visible (UV-vis) diffuse reflectance absorbance spectra (DRS) and electrochemical impedance spectra (EIS) studies clarified the enhanced photocatalytic efficiencies of CdS/GR nanocomposites. X-ray diffraction (XRD) analysis and photoelectrochemical curves both proved that the nanocomposites could suppress the photocorrosion of CdS NCs in the presence of graphene sheets.

\section{Experimental}

\subsection{Preparation of the CdS/GR nanocomposites}

A series of CdS/GR nanocomposites with various graphene contents were synthesized as in our previous work [17-19]. Briefly, $17 \mathrm{mg}$ grapheme oxide (GO) sheets (prepared by the modified Hummers' method) were dispersed in $10 \mathrm{~mL}$ twice-distilled water by sonication for $30 \mathrm{~min}$ to give a brown solution, and $50 \mathrm{~mL} 0.035 \mathrm{~mol} / \mathrm{L} \mathrm{Cd}\left(\mathrm{NO}_{3}\right)_{2}$ solution was added drop-by-drop into the as-prepared solution under stirring for 3 h. Then $\mathrm{H}_{2} \mathrm{~S}$ gas was bubbled through this dispersion for $1 \mathrm{~h}$ to form a green CdS/GR precipitation in situ. The resulting solids were centrifuged and washed three times with distilled water and acetone, and dried in vacuum at $45^{\circ} \mathrm{C}$ for $24 \mathrm{~h}$. In addition, CdS/GR nanocomposite doped with different graphene amounts were prepared by the above procedure with various amounts of GO sheets and free CdS NCs was obtained in the absence of GO sheets.

\subsection{Characterization of the CdS/GR nanocomposites}

Transmission electron microscopy (TEM) image was obtained with a JEOL 2100 transmission electron microscope (JEOL, Japan) operated at $200 \mathrm{kV}$. The UV-vis DRS of the samples were measured using a Perkin-Elmer Lambda 18 UV-visNIR spectrometer. XRD analysis was conducted using a Bruker D8 diffractometer with high-intensity $\mathrm{Cu} K_{\alpha}(\lambda=1.54 \AA$ A $)$ radiation. The visible-light source was a 250-W Xe lamp (Beijing Trusttech Co., Ltd.) with an intensity (passing through a 400-nm UV-cut filter) of $100 \mathrm{~mW} / \mathrm{cm}^{2}$. All electrochemical and photoelectrochemical measurements were conducted using a CHI660 B electrochemical analyzer (Chen Hua Instruments, Shanghai, China) with a conventional three-electrode system, in which a glassy carbon electrode (GCE, $3 \mathrm{~mm}$ in diameter) was used as the working electrode, $\mathrm{Ag} / \mathrm{AgCl}$ was used as the reference electrode, and platinum wire was used as the counter electrode. The photoelectrochemical curves were obtained in $0.1 \mathrm{~mol} / \mathrm{L} \mathrm{Na}_{2} \mathrm{SO}_{4}$ at $0 \mathrm{~V}$ with a scan rate of $100 \mathrm{mV} / \mathrm{s}$. EIS (presented as Nyquist plots) was performed in a $0.1 \mathrm{~mol} / \mathrm{L} \mathrm{KCl} \mathrm{so-}$ lution containing $5 \mathrm{mmol} / \mathrm{L} \mathrm{Fe}(\mathrm{CN})_{6}^{3-/ 4-}$, across a frequency range from $100 \mathrm{kHz}$ to $0.05 \mathrm{~Hz}$ at $0.24 \mathrm{~V}$, and the amplitude of the applied sine wave potential in each case was $5 \mathrm{mV}$.

\subsection{Fabrication of modified electrodes}

Prior to modification, the GCE was polished with sand paper and then with 1.0, 0.3, and $0.05 \mu \mathrm{m}$ alumina slurries, and sonicated in a water bath to remove any residues. CdS/GR nanocomposite (1 mg) with $4.6 \mathrm{wt} \%$ graphene sheets (denoted by CdS/GR-4.6\%) was dispersed in $1 \mathrm{~mL}$ of twice-distilled water to produce a CdS/GR-4.6\% homogeneous suspension, and then $6 \mu \mathrm{L}$ of the suspension were cast on the pretreated GCE surface and dried in air at room temperature to form a GCE modified with CdS/GR-4.6\% nanocomposite (denoted by CdS/GR-4.6\%/ GCE). As a comparison, CdS/GCE and blend/GCE (a physical mixture of graphene sheets and CdS NCs with a graphene sheet content of $4.6 \mathrm{wt} \%$ ) were prepared using similar procedures.

\subsection{Photocatalytic measurements}

The photocatalytic activities of the as-prepared CdS/GR nanocomposites were investigated using the photodegradation of MB under visible-light irradiation. A 250-W Xe lamp was used as the visible-light source. A UV-cut filter, which can filter out UV light with wavelengths below $400 \mathrm{~nm}$, was placed between the glass tube and the lamp. The CdS NCs and the blend 
(physical mixture of graphene sheets and CdS NCs with a graphene sheet content of $4.6 \mathrm{wt} \%$ ) were used as reference photocatalysts. Before illumination, the catalysts $(10 \mathrm{mg})$ were dispersed in MB $(100 \mathrm{~mL}, 10 \mathrm{mg} / \mathrm{L}$, from Shanghai Chemical Company) by sonication to obtain a suspension; the suspension was stirred in the dark to establish an adsorption/desorption equilibrium. Then the incandescent lamps were opened. During the photodegradation, the temperature was kept at $25{ }^{\circ} \mathrm{C}$ and constantly air-equilibrated under stirring. Every $0.5 \mathrm{~h}, 3 \mathrm{~mL}$ of suspension were centrifuged to remove particles. The filtrates were analyzed by recording the changes in the maximum absorption band for MB (664 nm). The degradation efficiency of $\mathrm{MB}$ was calculated as degradation efficiency = $\left[\left(A_{\text {Init }}-A_{\text {Test }}\right) / A_{\text {Init }}\right] \times 100 \%$, where $A_{\text {Init }}$ and $A_{\text {Test }}$ represented the absorbances of the initial (without treatment) and test MB samples, respectively.

To evaluate the photostabilities of the photocatalysts, the CdS/GR-4.6\% nanocomposite was separated from the suspension after running the first photocatalytic degradation reaction for $3 \mathrm{~h}$, washed with water, and dried at $50{ }^{\circ} \mathrm{C}$. The recovered photocatalysts were used for the following run of the photocatalytic degradation reaction under the same conditions [14].

\section{Results and discussion}

\subsection{Evaluation of photocatalytic efficiency}

\subsubsection{Photocatalytic efficiencies of CdS/GR nanocomposites}

The photocatalytic efficiencies of the CdS/GR nanocomposites were measured using the photodegradation of MB under visible light $(\lambda>400 \mathrm{~nm})$; the results are shown in Fig. 1(a). Without any photocatalyst, MB was hardly self-photodegraded. The photocatalytic efficiencies on addition of photocatalysts increased greatly as a result of the integration of semiconductor nanomaterials. Furthermore, the photocatalytic efficiencies of various CdS/GR samples were greater than that of the CdS NCs. Notably, the photocatalytic efficiency of the CdS/GR-4.6\% photocatalyst was twice that of the blend. This implied that there was no interaction between the CdS NCs and graphene sheets in the physical mixture. The CdS/GR-4.6\% photocatalyst

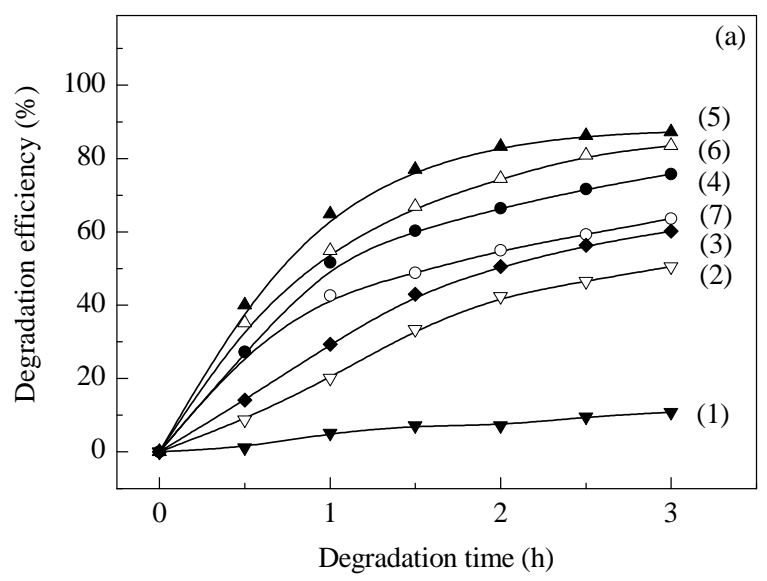

had the best photocatalytic efficiency, 2.3 times that of CdS NCs. The enhanced photocatalytic efficiency of the CdS/GR nanocomposites could be ascribed to electronic interactions and inter-electron transfer at the interfaces between the CdS NCs and graphene sheets [6]. The photoreaction process was fitted to pseudo-first-order kinetics [9], and the $k$ value is shown in Fig. 1(b). It can be seen that the photocatalytic efficiencies of the CdS/GR nanocomposites were influenced by the introduction of graphene sheets. As the graphene sheet content increased from 2.4 to $4.6 \mathrm{wt} \%$, the degradation rate $(k)$ increased from 0.46 to $0.74 \mathrm{~h}^{-1}$. However, once the graphene sheet content was relatively high ( $>4.6 \mathrm{wt} \%$ ), the photocatalytic rate then decreased with increasing graphene sheet content. At higher graphene sheet contents, graphene sheets accumulated on the CdS NCs surface might cause increased absorbance and scattering of photons [6], resulting in a decrease in the photocatalytic efficiency. The optimal graphene sheet content was approximately $4.6 \mathrm{wt} \%$. To achieve the best photoreaction response, the CdS/GR-4.6\% nanocomposite was therefore chosen as the photocatalyst in our experiments.

\subsubsection{UV-vis DRS and electrochemical analyses}

The UV-vis DRS of the CdS NCs and CdS/GR-4.6\% nanocomposite were used to further demonstrate the interactions between CdS NCs and graphene sheets. Compared with the CdS $\mathrm{NCs}$, there was an obvious red shift of ca. $50 \mathrm{~nm}$ in the absorption edge of the CdS/GR nanocomposite (Fig. 2(a)). The energy band gap $\left(E_{\mathrm{g}}\right)$ value of a semiconductor material can be calculated from the equation $(A h v)^{n}=C\left(h v-E_{\mathrm{g}}\right)$, where $h v$ is the photon energy, $A$ is the absorption coefficient, $C$ is a constant for the material, and $n$ is 2 for a direct transition or $1 / 2$ for an indirect transition [20]. The $E_{\mathrm{g}}$ values of the CdS NCs and CdS/GR nanocomposite were calculated from the observed UV-vis DRS and are shown in Fig. 2(b). The $E_{\mathrm{g}}$ values for the CdS NCs and CdS/GR nanocomposite were 2.38 and $2.26 \mathrm{eV}$, respectively. The change in the band gap values was ascribed to the incorporation of graphene sheets into the CdS NCs. The formation of chemical bonds between the CdS NCs and graphene sheets narrowed the band gap and extended the optical absorption range [5]. The visible-light response is therefore

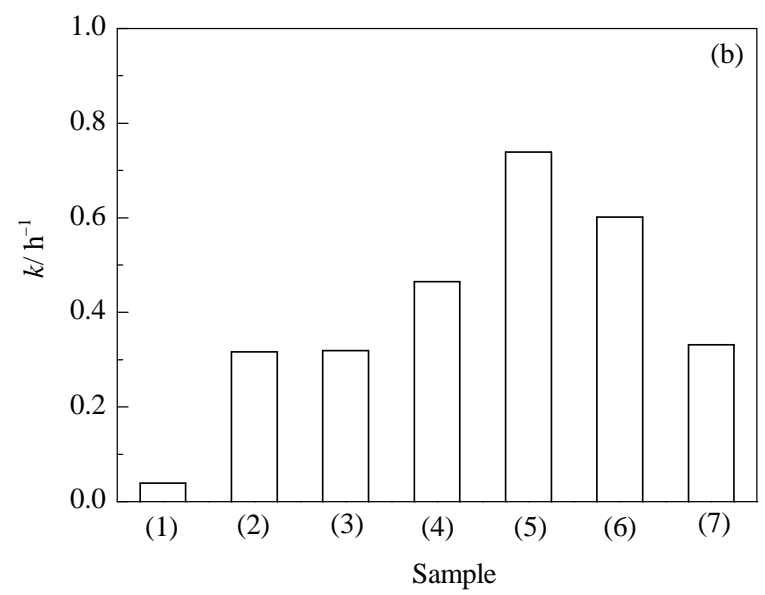

Fig. 1. Plots of degradation of MB (a) and the rate constant $k$ (b) over photocatalysts under visible-light irradiation. (1) No catalysts; (2) CdS NCs; (3) Physical mixture of CdS NCs and graphene sheets; (4) CdS/GR-2.4\%; (5) CdS/GR-4.6\%; (6) CdS/GR-10.7\%; (7) CdS/GR-30\%. 

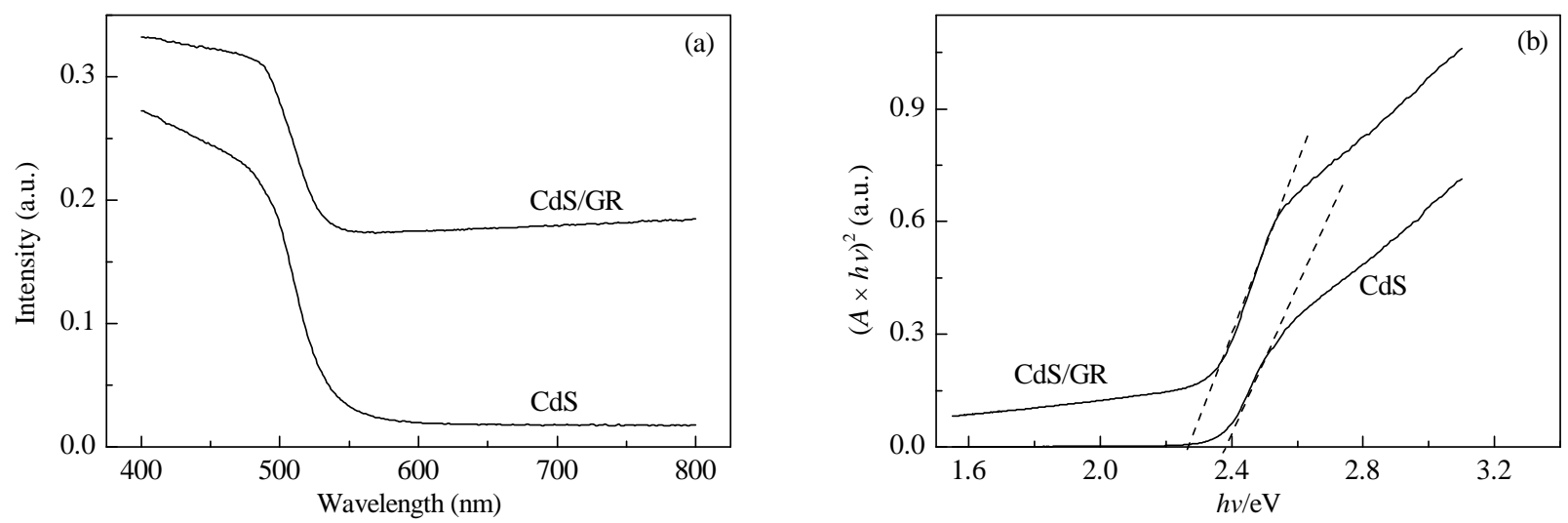

Fig. 2. The UV-vis diffuse reflectance absorption spectra (a) and plot of $(A \times h v)^{2}$ versus $h v$ (b) of CdS NCs and CdS/GR-4.6\% nanocomposites.

greater after the introduction of graphene into the CdS NCs.

EIS analysis is an important electrochemical method for studying interfacial charge transfer at electrode surfaces. To evaluate the electron-transporting ability of the CdS/GR-4.6\% nanocomposite, EIS of different electrodes were measured using $\mathrm{K}_{3} \mathrm{Fe}(\mathrm{CN})_{6} / \mathrm{K}_{4} \mathrm{Fe}(\mathrm{CN})_{6}$ as a redox probe. As shown in Fig. 3, the radius of the arc on the EIS of the CdS/GR-4.6\%/GCE was much smaller than those of the CdS/GCE and blend/GCE. Although the blend/GCE and CdS/GR-4.6\%/GCE had the same graphene sheet content, the arc radius on the EIS of CdS/ GR-4.6\%/GCE was $2.4 \mathrm{k} \Omega$, which was smaller than that of the blend/GCE, indicating that electronic interactions and inter-electron transfer at the interfaces between CdS NCs and graphene sheets [6] in the CdS/GR-4.6\% nanocomposite could facilitate interfacial charge transfer [9]. Each arc radius on the EIS of the modified electrodes under visible-light irradiation was smaller than that in the dark. These results show that more effective photogenerated electron-hole pair separation and faster interfacial charge transfer occurred on the modified electrodes under visible-light irradiation [21]. Overall, the graphene sheets in the nanocomposites acted as electron-acceptors and enabled interfacial charge transfer, thereby inhibiting charge recombination, reducing photocorrosion, and enhancing the photocatalytic efficiency.

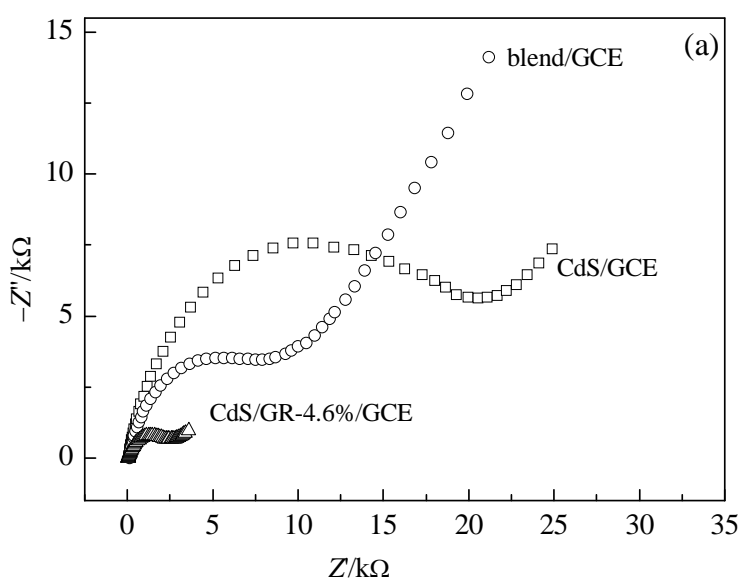

\subsection{Mechanism of enhanced photocatalytic efficiency}

Based on the above results and discussion, a schematic diagram of the mechanism of the photocatalytic and anti-photocorrosion processes over $\mathrm{CdS} / \mathrm{GR}$ photocatalysts is shown in Fig. 4. Under visible-light irradiation, the photogenerated electrons are transferred from the valence band to the conduction band of CdS in the CdS/GR nanocomposite, generating holes in the valence band [21]. The photogenerated electrons and holes move in opposite directions. Graphene, acting as an excellent electron acceptor and transporter [22,23], improves the interfacial electron transfer and restrains recombination of the electron-hole pairs. The reduced recombination probability and efficient charge separation lead to improved photocatalytic efficiency [5]. Concurrently, photogenerated electrons rapidly transferring from $\mathrm{CdS}$ to the graphene sheets successfully suppress charge recombination. The holes generated in the valence band of $\mathrm{CdS}$ react with $\mathrm{OH}^{-}$to form hydroxyl radicals $(\cdot \mathrm{OH})$, which have a high potential for the photodegradation of $\mathrm{MB}$ [24-26]. The MB, which is adsorbed on the graphene surface by $\pi-\pi$ stacking until adsorption/desorption equilibrium [27], is photodegraded to $\mathrm{CO}_{2}, \mathrm{H}_{2} \mathrm{O}$, and other organic micromolecules.

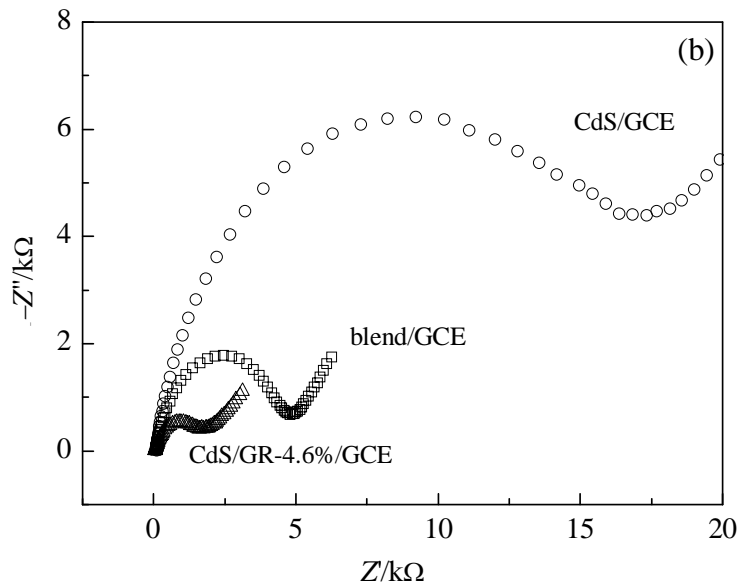

Fig. 3. (a) EIS of CdS/GCE, blend/GCE, and CdS/GR-4.6\%/GCE before visible-light irradiation; (b) EIS of CdS/GCE, blend/GCE, and CdS/GR-4.6\%/GCE under visible-light irradiation in $0.1 \mathrm{~mol} / \mathrm{L} \mathrm{KCl}$ in the presence of $5 \mathrm{mmol} / \mathrm{L} \mathrm{Fe}(\mathrm{CN})_{6} 3^{3-/ 4-}$ at $0.24 \mathrm{~V}$. 


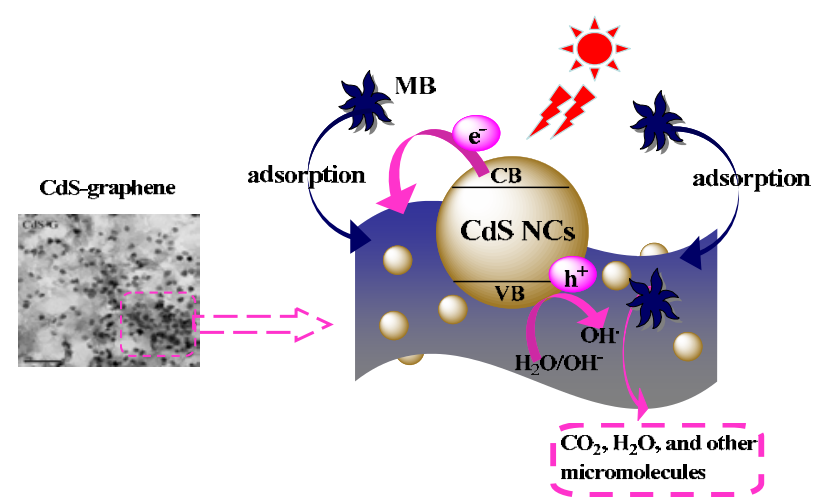

Fig. 4. The mechanism scheme of photocatalysis process under visible-light irradiation.

\subsection{Inhibition of photocorrosion behavior}

\subsubsection{Photostability of CdS/GR-4.6\% nanocomposite}

In "green chemistry", the catalytic reactions used and the recovery and reuse of catalysts are important factors in terms of the stringent ecological and economic demands for sustainability [28]. To evaluate the photostability of the CdS/GR-4.6\% photocatalyst, recycling tests were performed for the CdS NCs and CdS/GR-4.6\% nanocomposite in the photodegradation of MB. The results are shown in Fig. 5. As can be seen, the photocatalytic efficiency of the CdS NCs was poor, much lower than that of the CdS/GR-4.6\% nanocomposite, indicating that the distinctive structure of the graphene sheets is critical for photogenerated carrier separation. Recycling tests for the CdS/ GR-4.6\% nanocomposite were conducted in five consecutive runs. It was found that there was no significant decrease in photodegradation rate during the five continuous cycles. The results demonstrated not only the good stability but also the excellent anti-photocorrosion behavior of the CdS/GR-4.6\% photocatalyst.

\subsubsection{XRD patterns of CdS/GR-4.6\% nanocomposite}

The suppression of photocorrosion by the CdS/GR nanocomposites was also confirmed by XRD; the results are shown in Fig. 6. The CdS/GR nanocomposites had a crystalline structure similar to that of the CdS NCs before photodegradation.

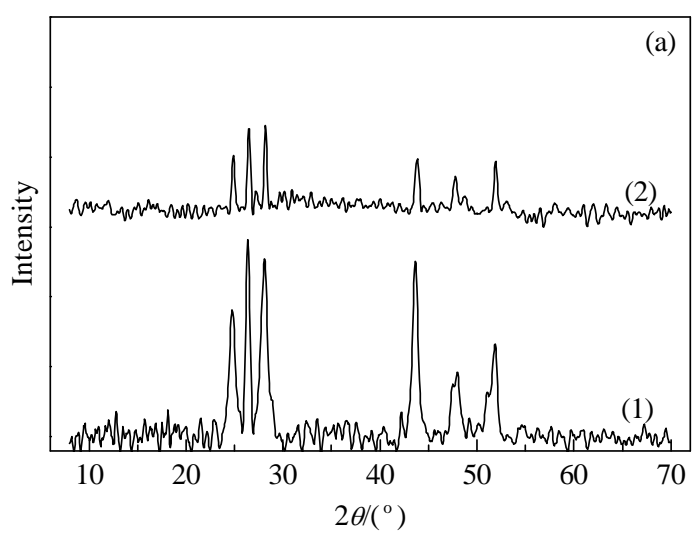

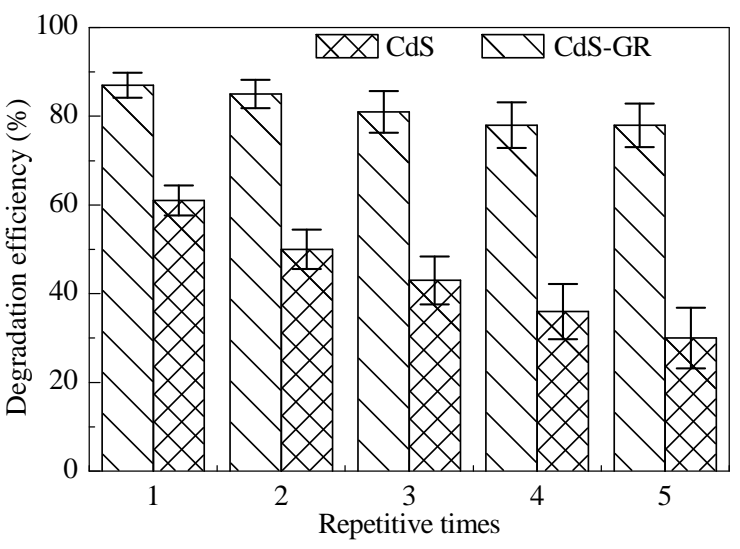

Fig. 5. Cycling runs in the photodegradation of MB in the presence of CdS/GR-4.6\% under visible-light irradiation.

The characteristic diffraction peak of graphene at $23^{\circ}$ could not be detected for the CdS/GR nanocomposites because of the low diffraction intensity and low amounts of graphene in the nanocomposites [5,29]. Figure 6(a) shows that the crystalline structure of the CdS NCs was destroyed after the photocatalytic process, which indicated that the CdS NCs had been photocorroded. In contrast, there were no significant differences between the XRD patterns of the CdS/GR nanocomposites before and after the photocatalytic process (Fig. 6(b)). The results further indicated that the graphene sheets could prevent photocorrosion of the CdS NCs and enhance the CdS NC stability during photoreactions.

\subsubsection{Photoelectrochemical activity of CdS/GR-4.6\% nanocomposite}

Photoelectrochemical experiments were performed to investigate the electronic interactions between the CdS NCs and graphene sheets. Figure 7 shows the photocurrent responses for CdS/GCE, blend/GCE, and CdS/GR/GCE. CdS/GR/GCE exhibited the highest photoelectrochemical activity, and the activity was 2.2 times that of CdS/GCE. It was clear that fast and uniform photocurrent responses were observed for each switch-on and switch-off event in every electrode. This photoresponsive phenomenon was entirely reversible. When the illumination was stopped, the photocurrent decreased back to

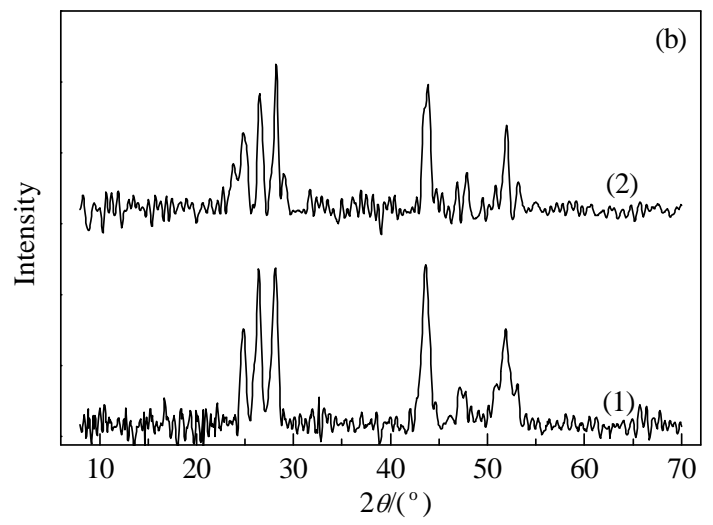

Fig. 6. XRD patterns of CdS NCs (a) and CdS/GR-4.6\% nanocomposites (b) before (1) and after (2) photodegradation of MB under visible-light irradiation. 


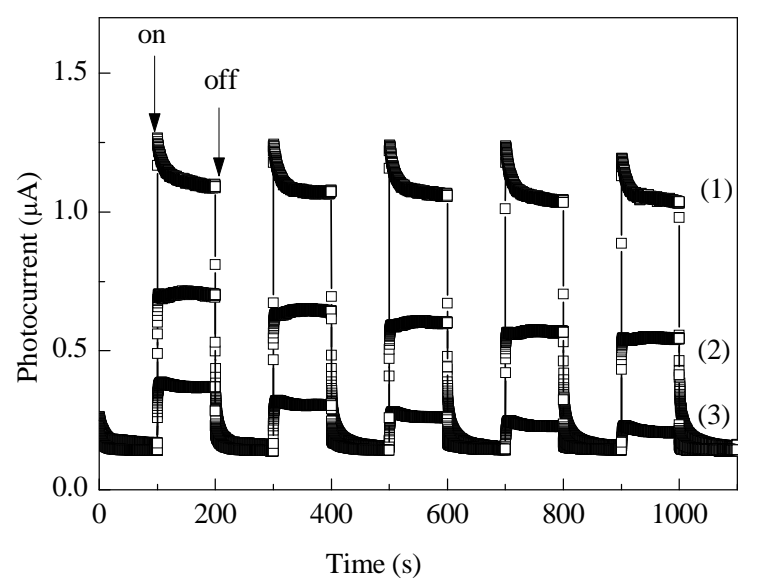

Fig. 7. Photocurrent graphs of CdS/GR-4.6\%/GCE (1), blend/GCE (2), and CdS/GCE (3) in $0.1 \mathrm{~mol} / \mathrm{L} \mathrm{Na}_{2} \mathrm{SO}_{4}$ at $0 \mathrm{~V}$.

zero. These results are attributed to fast transfer of the photoinduced carriers and a low recombination rate of electronhole pairs [15] in the CdS/GR nanocomposites. In contrast, the photocurrent responses of CdS/GCE and blend/GCE gradually decreased because of the instability and photocorrosion of the CdS NCs. The CdS/GR nanocomposites were photostable and experienced little photocorrosion after doping with graphene sheets. These results were in good agreement with the XRD patterns and recycling tests.

\section{Conclusions}

A series of CdS/GR nanocomposites with various graphene contents were successfully synthesized to investigate their photocatalytic efficiencies and photostabilities under visible-light irradiation. The photocatalytic efficiencies of the as-prepared CdS/GR nanocomposites achieved a maximum at a graphene content of $4.6 \mathrm{wt} \%$. The introduction of graphene sheets improved the migration efficiency of photogenerated carriers and inhibited charge carrier recombination. The results proved that the photocorrosion of CdS NCs was effectively suppressed by the introduction of graphene sheets. The present investigation highlights the importance of not only the photocatalytic efficiency of a photocatalyst but also its anti- photocorrosion behavior and photostability. It is hoped that our current work will enable the efficient use of graphenebased semiconductor nanocomposites as visible-light photocatalysts.

\section{References}

[1] Huang C C, Li C, Shi G Q. Energy Environ Sci, 2012, 5: 8848

[2] An X Q, Yu J C. RSC Adv, 2011, 1: 1426

[3] Zhang H, Lv X J, Li Y M, Wang Y, Li J H. ACS Nano, 2010, 4: 380

[4] Zhang Y H, Tang Z R, Fu X Z, Xu Y J. ACS Nano, 2011, 5: 7426

[5] Li Q, Guo B D, Yu J G, Ran J R, Zhang B H, Yan H J, Gong J R. J Am Chem Soc, 2011, 133: 10878

[6] Xu T G, Zhang L W, Cheng H Y, Zhu Y F. Appl Catal B, 2011, 101: 382

[7] Wang X W, Tian H W, Yang Y, Wang H, Wang S M, Zheng W T, Liu Y C. J Alloys Compd, 2012, 524: 5

[8] Shylesh S, Schünemann V, Thiel W R. Angew Chem Int Ed, 2010, 49: 3428

[9] Zhang H, Zhu Y F. J Phys Chem C, 2010, 114: 5822

[10] Zong X, Yan H J, Wu G P, Ma G J, Wen F Y, Wang L, Li C. J Am Chem Soc, 2008, 130: 7176

[11] Wu Z Y, Zhao G H, Zhang Y N, Tian H Y, Li D M. J Phys Chem C, 2012, 116: 12829

[12] Fang Z, Liu Y F, Fan Y T, Ni Y H, Wei X W, Tang K B, Shen J M, Chen Y.J Phys Chem C, 2011, 115: 13968

[13] Ma L L, Sun H Z, Zhang Y G, Lin Y L, Li J L, Wang E, Yu Y, Tan M, Wang J B. Nanotechnology, 2008, 19: 115709

[14] Peng T Y, Li K, Zeng P, Zhang Q G, Zhang X G. J Phys Chem C, 2012, 116: 22720

[15] Zhang N, Zhang Y H, Pan X Y, Fu X Z, Liu S Q, Xu Y J. J Phys Chem C, 2011, 115: 23501

[16] Vinod K N, Puttaswamy, Gowda K N N. Ind Eng Chem Res, 2010, 49: 3137

[17] Wang K, Liu Q, Wu XY, Guan Q M, Li H N. Talanta, 2010, 82: 372

[18] Wang K, Liu Q, Guan Q M, Wu J, Li H N, Yan J J. Biosens Bioelectron, 2011, 26: 2252

[19] Wang K, Liu Q, Dai L N, Yan J J, Ju C, Qiu B J, Wu X Y. Anal Chim Acta, 2011, 695: 84

[20] Tauc J, Menth A.J Non-Cryst Solids, 1972, 8-10: 569

[21] Wang K, Wu J, Liu Q, Jin Y C, Yan J J, Cai J R. Anal Chim Acta, 2012, 745: 131

[22] Shao Y Y, Wang J, Wu H, Liu J, Aksay I A, Lin Y H. Electroanalysis, 2010, 22: 1027

\section{Graphical Abstract}

\section{Chin. J. Catal., 2013, 34: 1876-1882 doi: 10.1016/S1872-2067(12)60677-9}

Visible-light photocatalytic efficiencies and anti-photocorrosion behavior of CdS/graphene nanocomposites: Evaluation using methylene blue degradation

Jiajia Yan, Kun Wang *, Hui Xu, Jing Qian, Wei Liu, Xingwang Yang, Huaming Li Jiangsu University

$\mathrm{CdS} /$ graphene nanocomposite with $4.6 \mathrm{wt} \%$ graphene sheets displayed the optimum photocatalytic efficiency for methylene blue degradation. They showed excellent anti-photocorrosion behavior and photostablity under visible-light irradiation.

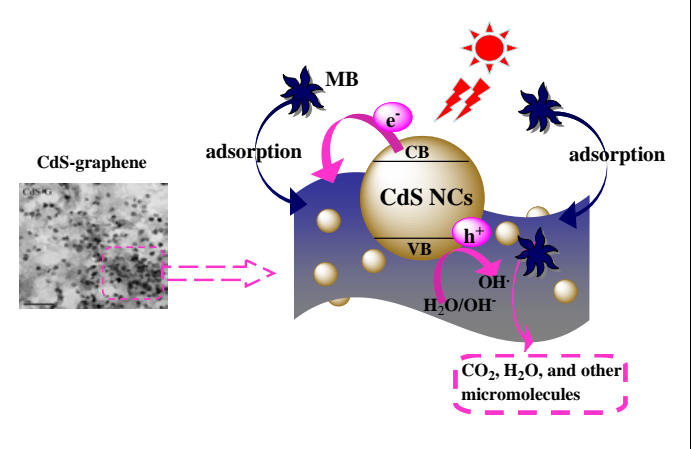


[23] Brownson D A C, Banks C E. Analyst, 2010, 135: 2768

[24] Tak Y J, Kim H Y, Lee D W, Yong K J. Chem Commun, 2008: 4585

[25] Lin G F, Zheng J W, Xu R. J Phys Chem C, 2008, 112: 7363
[26] Li XY, Hu C G, Wang X, Xi Y. Appl Surf Sci, 2012, 258: 4370

[27] Liu Z, Robinson J T, Sun X M, Dai H J. J Am Chem Soc, 2008, 130: 10876

\title{
$\mathrm{CdS} /$ 石墨烯纳米复合物的可见光催化效率和抗光腐蚀行为
}

\author{
严佳佳, 王 坤*, 许 晖, 钱 静, 刘 巍, 杨兴旺, 李华明 \\ 江苏大学化学化工学院现代农业设备与技术重点实验室, 江苏镇江212013
}

摘要: 制备了一系列CdS纳米晶/石墨烯(CdS/GR)复合物, 并在可见光照条件下评价了其光催化降解亚甲基蓝的光催化效率和抗 光腐蚀行为. 研究表明, 石墨烯的引入加速了CdS纳米晶(NCs)光生电子的迁移速率, 抑制了其光生电子-空穴的复合, 有效改善了 其光催化降解有机污染物的性能. CdS/GR复合物中的石墨烯含量显著影响其光催化效率, 其中石墨烯含量为 $4.6 \%$ 的光催化剂效 率最高, 其光电流是CdS NCs的2.3倍. 利用光电化学和X射线衍射技术进一步证实, 石墨烯的引入抑制了CdS NCs光腐蚀的发生, 提高了CdS/GR复合物的光催化稳定性.

关键词: 硫化镉纳米晶; 石墨烯纳米复合物; 抗光腐蚀; 光催化; 亚甲基蓝降解

收稿日期: 2013-02-21. 接受日期: 2013-04-01. 出版日期: 2013-10-20.

*通讯联系人. 电话: (0511)88791800; 传真: (0511)88791708; 电子信箱: wangkun@ujs.edu.cn

基金来源：国家自然科学基金(21175061, 21007021); 现代农业设备与技术重点实验室项目(NZ201109); 中国博士后科学基金 (2012M520998).

本文的英文电子版由Elsevier出版社在ScienceDirect上出版(http://www.sciencedirect.com/science/journal/18722067). 